\title{
Domesticar la violencia contra las mujeres, una forma de desactivar el conflicto intergéneros
}

\author{
Begoña MARUGÁn PINTOS \\ Departamento de CC. Política y Sociología de la Universidad Carlos III de Madrid. \\ bmarugan@polsoc.uc3m.es
}

Recibido: 15.09 .2012

Aceptado: 30.09 .2012

\begin{abstract}
RESUMEN
La violencia contra las mujeres ha sido referenciada bajo distintas denominaciones: «maltrato», «violencia doméstica», «machista», «de género», «terrorismo doméstico», etc. Estas distintas denominaciones han significado una ampliación o una reducción de los comportamientos que en cada momento se consideraban violentos. Este artículo analizará el camino conceptual por el que ha transitado la violencia sobre las mujeres, en el Estado español, a lo largo de las últimas décadas. A través de este proceso de reconstrucción nominal se observará un doble movimiento: primero se irá ampliando progresivamente el tipo de conductas que exceden lo socialmente admitido, para, posteriormente, encorsetarla como «violencia doméstica» o «violencia en los entornos familiares», lo que no hace sino ocultar que en las relaciones de dominación que mantienen los hombres sobre las mujeres radica la raíz del problema.
\end{abstract}

Palabra clave: violencia doméstica, violencia contra las mujeres, violencia de género, dominación masculina, ideología de la domesticidad.

\section{"Domesticating" Violence: A Way to Refusing the Intergender Conflict}

\begin{abstract}
Violence against women has been referred to by different names: «abuse», «domestic violence», «sexist violence», «gender violence», «domestic terrorism», etc. These denominations have pointed to an extension or a reduction of the behaviors that were considered to be violent in each particular period. This article will explore the conceptual changes which have taken place concerning violence against women in the Spanish State, over the last few decades. Through this process of nominal reconstruction a double movement can be observed: firstly, the type of conduct considered outside of the socially acceptable was progressively expanded, only later to restrict this behavior as «domestic violence» or «violence in family environments», all of which did nothing but hide the ultimate root of the problem: the domination of men over women.
\end{abstract}

Keywords: Domestic violence, violence against women, gender violence, male domination, ideology of domesticity.

\section{INTRODUCCIÓN}

El Diccionario de Sociología define la «violencia» como una interacción social en la cual una persona o cosa resultan dañada de manera intencional o sobre la cual recae 
la amenaza creíble de padecer quebranto (Reinares,1998:820). Una pensadora tan ligada al estudio de los orígenes de los conceptos políticos como Hannah Arendt recuerda que la violencia se utiliza siempre con un carácter instrumental, con un fin de dominación. A partir de una aproximación semántica, Corsi (Corsi, 1995:23) focaliza sus observaciones en las conductas violentas individuales. Estas se utilizan para la resolución de conflictos interpersonales en un intento de doblegar la voluntad del otro, de anularlo precisamente en su calidad de «otro». La violencia busca eliminar los obstáculos que se oponen al propio ejercicio del poder, mediante el control de la relación obtenido a través del uso de la fuerza. Hablar de violencia supone reconocer la existencia de formas de ejercicio de poder e implica la existencia de situaciones de desigualdad reales y/o simbólicas.

Partir de la denominación de violencia implica admitir la existencia de conductas relacionales que rebasan el nivel de lo aceptable. García Selgás aplica esta conceptualización a la violencia de género para «designar a aquellas conductas que rebasan las convenciones que regulan lo "normal" o "aceptable" dentro de las relaciones de pareja heterosexuales» (García Selgás, 2009:58). Ahora bien, este umbral de lo aceptable varía en el tiempo y en el espacio y por ello en este artículo se tratará de mostrar cómo los niveles de tolerancia de determinados comportamientos tanto en la sociedad como en las parejas, se han ido modulando a lo largo de la historia y dando lugar a diferentes denominaciones hasta llegar a la designación de «violencia doméstica».

Para aquellas personas que sabemos que los intercambios simbólicos encubren y reflejan relaciones de poder (Bourdieu,1982) y la sociedad se reproduce simbólicamente mediante un sistema de símbolos y significantes que transmiten una concepción del mundo, desvelar lo que se ilumina y se oculta en cada una de las denominaciones de la «violencia contra las mujeres» hasta llegar a la «violencia doméstica» se presenta como una cuestión vital. Al rastrear la construcción de los distintos significantes atribuidos a este delito se observa una progresiva ampliación del campo visual y de las agresiones que padecen las mujeres hasta llegar a la denominación de «violencia contra las mujeres», sin embargo, a finales de los años noventa ésta se vio restringida a la que tiene lugar en el espacio doméstico. El calificativo «doméstica» no hace sino silenciar la relación de dominación que mantienen los hombres sobre las mujeres al desdibujar el conflicto político-social latente y reducir la violencia que se ejerce sobre las mujeres a una cuestión del ámbito familiar que afecta sólo a determinadas parejas. 


\section{PROCESO DE AMPLIACIÓN DEL CAMPO SEMÁNTICO Y DE LAS CONDUCTAS AGRESIVAS SOBRE LAS MUJERES}

Los movimientos feministas fueron los encargados de visibilizar, en la década de los setenta, el fenómeno de la violencia sobre las mujeres. Las primeras denuncias públicas se formularon por las inglesas y americanas al demandar el derecho al propio cuerpo $^{1}$ y hacer público su rechazo de las violaciones. La violación es una acto de violencia cuya razón de ser es la humillación y la víctima se siente tratada como un objeto.

En esta labor de denuncia el feminismo contó con el apoyo de la investigación social que mostraba tanto la gravedad del delito, como la complicidad social con la que el mismo contaba. "La violación es un crimen de poder, no de placer» (Browmiller, 1975) que ataca física y sexualmente a la víctima. A la violada se le niega el derecho básico de disponer de su propio cuerpo, pero además, se le acusa de ser la instigadora del delito y se le exige demostrar su resistencia al mismo (BurgesS y Holstram, 1974) $)^{2}$.

La imagen del violador que se manejaba entonces era la de un agresor perturbado ajeno a la víctima, a la que asaltaba por la noche, en sitios inhóspitos. Sin embargo, en 1983-1984, la Asociación Women Against Rape investigó la prevalencia de violaciones en el centro de Londres y concluyó que el 10\% de las encuestadas habían sido violadas en su hogar. Es entonces cuando se empiezan a visibilizar la existencia de malos tratos en los entornos familiares, donde además de la existencia de violaciones, en ocasiones estas se acompañan de malos tratos por parte de la pareja.

Así, este tipo de conductas abusivas e intolerables por parte de los varones que las feministas venían denunciando empiezan a ser reconocidas institucionalmente. En la IV Conferencia Mundial sobre la Mujer, celebrada en Copenhague en 1980, se constataba que «la violencia contra las mujeres en el entorno familiar era el crimen encubierto más frecuente en el mundo», pero se atribuía su origen a motivos coyunturales (aislamiento geográfico y social, dificultades económicas, irregularidad

\footnotetext{
${ }^{1}$ Algunas de estas ideas aparecen en publicaciones como Nuestros cuerpos, nuestras vidas (1969) del Colectivo de Mujeres de Boston, todo un clásico, reeditado en España, en el año 2000, por Plaza y Janes.

${ }^{2}$ Lo que tuvo su plasmación en un número importante de sentencias en España en los años ochenta, como la famosa de la minifalda ó aquella otra en la que los violadores fueron absueltos porque se acusó a la violada de «llevar una vida licenciosa» y probablemente subyace en las declaraciones de Todd Akin, político estadounidense republicano que, en plena campaña del candidato republicano Mitt Romney a la presidencia, afirmó que las víctimas de una violación "legítima" no suelen quedarse embarazadas. Según Akin, «si se trata de una violación legítima, el cuerpo de la mujer tiene mecanismos para cerrarse del todo y prevenir el embarazo» (El País, 20 de agosto de 2012) http://internacional.elpais.com/internacional/2012/08/20/actualidad/1345480654_857422.html (Fecha de consulta: 22 de agosto de 2012).
} 
del empleo, abuso de alcohol y de drogas, inseguridad, sentimiento de inferioridad e inestabilidad de las mujeres) que se expresan en individuos concretos. La ausencia de perspectiva social se reforzaba a través de una investigación predominantemente psiquiátrica y psicológica ${ }^{3}$. Se buscaba describir tanto el perfil de los agresores y de las víctimas (Ferreira, 1992), como «el ciclo de la violencia» (Walter, 1979).

A partir de la constatación de que los malos tratos no se producían únicamente en los países del Tercer Mundo se constituyen los primeros gabinetes jurídicos y psicológicos de mujeres. La experiencia de las feministas inglesas que habían abierto una casa refugio de mujeres golpeadas sirvió de modelo a otros países. En España, el movimiento feminista avanzaba en el camino de la reivindicación del cuerpo, con campaña por los anticonceptivos y el derecho al aborto en su batalla por hacer de lo personal algo político y demandaba al Estado programas concretos de atención para las víctimas de violencia sexual y de malos tratos. Una labor a la que contribuiría la creación del Instituto de la Mujer, en 1983.

La respuesta institucional se vio reforzada sinérgicamente por un movimiento feminista organizado en barrios y pueblos que solicitaba la tipificación de la violencia contra las mujeres como delito dentro del Código penal. La inclusión del artículo 425 del «maltrato por el esposo», en el año 1989 y las aportaciones desde el derecho (Rodríguez Ramos-Álvarez y García-Gómez Pavón), la criminología (Elena Larrauri) o la sociología (Miranda López, Fernández Villanueva) permitieron modificar la mirada psicológica anterior y buscar la raíz del problema en el universo cultural de la sociedad patriarcal. El origen de la violencia física y sexual, como la psicológica, la económica y la simbólica contra las mujeres estaba en los valores patriarcales (Miranda, 2002), según los cuales existe una intocable jerarquía de poder y dominación de los fuertes -los hombres adultos- sobre los débiles -las mujeres y los niños- (Gelles y Strauss, 1988).

En solo dos décadas se había pasado a hablar de la violación a los malos tratos físicos y de estos a la violencia contra las mujeres en sentido amplio. En 1992, el Consejo Económico y Social de la ONU define violencia contra la mujer como «todo acto de violencia basado en la pertenencia al sexo femenino que tenga, o pueda tener como resultado un daño o sufrimiento físico, sexual o psicológico para las mujeres, inclusive las amenazas de tales actos, la coacción o la privación arbitraria de libertad, tanto si se producen en la vida pública o privada» (art. 1 de la Declaración sobre la Eliminación de la Violencia Contra la Mujer de la ONU) y en 1995, en la Convención de Beijing, se explica que la violencia es «la manifestación de las

\footnotetext{
${ }^{3}$ En 1974 se publicó un notable estudio epidemiológico basado en entrevistas a 146 mujeres violadas que describieron con detalle lo que se llamó «síndrome del trauma de la violación» y en 1980, el Manual Estadístico de los Trastornos Mentales, en su tercera edición, reconoció el Estrés Postraumático a través de los veteranos de Vietnam y las mujeres violadas (Dio Bleichmar,2001)
} 
relaciones de poder históricamente desiguales entre mujeres y hombres, que han conducido a la dominación de la mujer por el hombre, la discriminación contra la mujer y a la interposición de obstáculos contra su pleno desarrollo». Con estos precedentes, la Resolución de la Comisión de Derechos Humanos 1997/44 de la ONU, exige la eliminación de la violencia contra las mujeres y exhorta a los Estados a «establecer y reforzar en la legislación nacional sanciones penales, civiles, laborales y administrativas para castigar y reparar los agravios infligidos a las mujeres y las niñas que sean objeto de cualquier forma de violencia, ya sea en el hogar, en el lugar de trabajo, la comunidad o la sociedad».

A partir de las declaraciones de la ONU, el Parlamento Europeo aprobó una serie de Resoluciones, entre ellas la A4-0250/97, sobre una campaña europea sobre tolerancia cero ante la violencia contra las mujeres. En esta Resolución se emplea por primera vez el término «violencia doméstica».

Y esto se produce el mismo año en que, en España, la televisión ofrece la entrevista con Ana Orantes confesando los maltratos de su ex esposo. El que juró venganza. Al día siguiente a la emisión de aquel programa Orantes fue quemada viva por su ex compañero y nuevamente la televisión reproducirá la entrevista detonante del asesinato.

Durante los dos meses siguientes el periódico de referencia dominante, El País, cita en primera plana los casos de asesinatos a mujeres que se van produciendo, de modo que la violencia doméstica se convierte en fenómeno mediático. Los medios de comunicación «redescubren la violencia». El protagonismo de los medios a partir de 1998 es incuestionable, pues éstos han jugado un papel fundamental en la dramatización -en el sentido que Goffman (1974) atribuye a este término- de los hechos violentos al conseguir conformar un público para la violencia que atenta contra las mujeres, pero esta vez reduciendo la misma únicamente a una de sus manifestaciones: la violencia doméstica.

\section{LA UTILIZACIÓN DEL TÉRMINO "VIOLENCIA DOMÉSTICA" Y SUS CONSECUENCIAS}

Los programas de televisión y reality shows en los que aparecían mujeres denunciando a sus parejas y la publicación constante de asesinatos de mujeres por parte de sus maridos o ex-maridos en los medios de comunicación contribuyeron decididamente a convertir este problema en un escándalo. El «bombardeo» mediático mostraba que las agresiones a las mujeres en los núcleos familiares no eran algo anecdótico, raro e infrecuente. La evolución del número de denuncias de malos tratos 
-a pesar de ser sólo un dato indicativo que mostraba el «iceberg» del problema ${ }^{4}$ - que aportaba el Ministerio de Interior no dejaba duda alguna ${ }^{5}$.

\subsection{LA POTENCIALIDAD DEL TÉRMINO}

Caracterizar la violencia como doméstica «expresaba muy bien la vulnerabilidad de las mujeres en el ámbito de mayor confianza y aplicaba la consigna "lo privado es público", tan querida al movimiento feminista» (Miranda, 2006:2486). Se transformó la percepción tradicional de que el maltrato era un problema de la víctima, y por ende, privado, para reconocer que el problema era del maltratador y por tanto de la comunidad. Al ser una cuestión de todos se requería de la actuación colectiva en todos los ámbitos y entre otros de la justicia penal. Y de ahí que durante unos años las propuestas institucionales apostaran hacia el recrudecimiento del castigo para los culpables $^{6}$ (Fortune y Adams, 2005:2504). En el cambio de la consideración del maltrato de «vicio» privado a delito semipúblico se observa la ruptura de la concepción dicotómica de los espacios, pues aunque el maltrato se cometiera en el hogar, como tal falta o delito, el Ministerio Fiscal tiene la obligación de perseguirlo. $\mathrm{Si}$ en otros tiempos la violencia era una herramienta de dominación masculina incuestionable ahora se convierte en un exceso, una interferencia en el orden de la reproducción de la sociedad actual.

Por otra parte, calificar la violencia como doméstica suponía renunciar abiertamente -cuando las mujeres ya lo habían hecho- a la ideología de la domesticidad que se impuso a mediados del siglo pasado. Esta ideología de la domesticidad consiste en una idealización y promoción de lo doméstico como ámbito de realización, desarrollo e influencia femenino ajeno al resto de ámbitos y con una cultura propia. Aunque esta idealización tuvo un particular reflejo en la vivencia del trabajo doméstico se extendió a todos los ámbitos de la vida y de modo muy especial

${ }^{4}$ Para dimensionar de modo más preciso el fenómeno se empezaron a realizar una serie de macro encuestas. La primera, se llevó a cabo en 1999, por Sigma Dos para el Ministerio de Trabajo y Asuntos Sociales. Los resultados de la misma cuantificaban en el 4,2\% la proporción de las mujeres españolas de más de 18 años que declaraban haber sido víctimas de malos tratos. Este porcentaje representaba un total de 640.000 mujeres entre las 15.028 .000 de esa edad. Por otra parte, un $12,4 \%$ de encuestadas fueron consideradas «técnicamente maltratadas», lo que suponía que el maltrato técnico afectaba a un total de 1.865 .000 mujeres.

${ }^{5}$ A esta información se puede añadir que según el $5^{\circ}$ Barómetro de Opinión, encargado por el Defensor del Pueblo y efectuado en enero de 1998, cinco millones y medio de personas afirmaban saber de algún caso de maltrato físico o psicológico a mujeres por parte de sus parejas.

${ }^{6}$ Entre los años 1999 y 2004, el Gobierno español impulsó una serie de iniciativas legislativas que han implicado cambios en el Código penal, ampliando su cobertura y endureciendo las penas. Se dio un giro penal porque se entendía que esta actuación conllevaba implícita una medida pedagógica, sin embargo, algunas juristas argumentaban que la aplicación del derecho penal por sí solo no era el instrumento para erradicar la violencia de género. El endurecimiento de las penas no tiene prácticamente transcendencia en el número de agresiones, pero sí lo tienen las medidas de alejamiento respecto a la seguridad de la víctima (Sánchez y Carrasco,2001:138). Quizá por esta razón, posteriormente, se aprobara la Ley 27/2003, de 31 de julio, reguladora de la Orden de Protección de las Víctimas de Violencia Doméstica. 
a la creación de una imagen femenina satisfecha y preocupada por la limpieza y bienestar de todos los miembros de la familia ${ }^{7}$. El que la esposa se dedicara exclusivamente a los cuidados del hogar y la familia era un elemento de prestigio toda vez que el funcionalismo parsoniano describía una ordenada organización social basada en la colaboración entre: los hombres, que representaban el rol instrumental como proveedores de los recursos económicos monetarios que las familias precisaban - y las mujeres, a las que correspondía el rol expresivo - encargadas de aportar el componente afectivo, de cuidado y atención a la familia -. Como responsables de mantener la armonía en los hogares las mujeres soportaban la dura carga de tener que mantener unida a la familia. Cualquier fisura en la pareja sería observada personal y socialmente como un fracaso en su papel. Sin embargo, los principios teóricos uniones basadas en el amor romántico y división de espacios y tareas entre los miembros del hogar- se desmoronaban ante la cruda realidad.

Los datos empíricos muestran que la familia, lejos de ser una institución ideal, de realización personal y comprensión recíproca, es uno de los espacios de conflicto por excelencia. «La familia, por sus características de intimidad, privacidad y creciente aislamiento, tiende a ser conflictiva. (...) Desde la investigación social Gelles y Strauss (1988) identificaron algunos factores como la cantidad de tiempo que los miembros permanecen juntos, la gama de actividades e interés, la intensidad del vínculo afectivo o el hecho que las decisiones de unos afecten a otros que pueden incrementar el riesgo potencial de una familia» (Corsi, 1995: 26-27). El componente afectivo y el mutuo conocimiento que existe entre todos los miembros hacen que cualquier disputa sea mucho más cruenta y dolorosa.

Por otra parte, los postulados de una ideal división del trabajo donde las mujeres «disfrutaban» del trabajo doméstico y de cuidados se había mostrado a todas luces falso y no sólo porque las mujeres dieran muestras de su rechazo a la «mística de la feminidad», sino porque la lógica de la empleabilidad individual bajo la cual se diseñaban las políticas de empleo europeo no tenían en cuenta las necesidades de las personas dependientes. Las crecientes exigencias de un mercado segmentado, desregulado, flexibilizado y precarizado ponen a las mujeres en posiciones extremadamente vulnerables. Las tensiones complejas y contradictorias que se generan entre la familia y el empleo complican la reproducción en los términos en los que se había venido dando de forma mayoritaria. Esta situación creó un grave problema de sobresaturación laboral a unas generaciones de mujeres que, cada vez más estresadas en sus dobles jornadas, no estaban dispuestas a soportar mucho más. No sólo la familia, también la propia convivencia estaba en juego. Se complica la

\footnotetext{
${ }^{7}$ Tras la Segunda Guerra Mundial, y una vez que los hombres volvieron de la contienda había a devolver a las mujeres al hogar. «El trabajo doméstico empezó a ser entendido como una "experiencia emocional”. Hacer la colada no era solo lavar la ropa, sino una expresión de amor, el ama de casa que amaba de verdad a su familia la protegía de la vergüenza del gris revelador» (Schwartz,2011:110).
} 
reproducción social en los términos en los que se estaba dando, las mujeres rechazan cada vez más su papel y la familia tradicional entra en crisis.

Ante esta evidencia el Estado interviene amortiguando y pacificando las tensiones existentes a favor de las mujeres y para ello les «promete» protección -seguridad e integridad - como parte de una política estratégica contra la violencia machista y reparto a través de las políticas igualitarias de conciliación. Una de las actuaciones se concretó en la aprobación de la Ley 39/1999 de Conciliación de la Vida Familiar y Laboral de las Personas Trabajadoras, aprobada el 5 de noviembre, trasposición tardía, lenta y contradictoria de las Directivas del Consejo 92/85/CEE, de 19 de octubre, y la 96/34/CE, del consejo, de 3 de junio, con la que pretende hacer más llevadera la vida familiar de las mujeres empleadas. La otra actuación estatal reseñable es su implicación en el tratamiento de la violencia doméstica.

\subsection{LIMITACIONES EN SU TRATAMIENTO}

Actualizada diariamente por los medios de comunicación como un suceso escandaloso ${ }^{8}$ impropio de un país democrático desarrollado, la violencia es asumida como competencia por el Estado. Por primera vez el Estado intentará limitar el poder de control de los cabezas de familia en los hogares, pero lo hará como si la violencia contra las mujeres fuera sólo una cuestión a gestionar y además la gestión se abordará como emergencia ${ }^{9}$. Gestionar la violencia como emergencia no permitiría acabar con la misma, ni siquiera contribuirá a paliar sus consecuencias, simplemente tratará de limitar las manifestaciones más brutales de la misma, que en este caso son los asesinatos y la violencia física producida por parte de los compañeros o ex compañeros sentimentales (Marugán y Vega, 2002). El Estado, en este sentido, normalizará el estado de excepción y reformulará su papel de forma creciente en términos de seguridad.

Periodistas y políticos, erigidos ahora en «especialistas» del debate, actuaban como dinamizadores del mismo, desplazando a un movimiento feminista que, como el resto de movimientos sociales, había cedido el protagonismo político. La violencia contra las mujeres, reducida ahora a violencia doméstica, se convirtió en una cuestión

\footnotetext{
${ }^{8}$ Lo cual semánticamente tuvo su reflejo en la denominación de este fenómeno como «terrorismo doméstico» que despertó la alarma social. En el contexto del 11 de septiembre se analizaron los efectos de la violencia doméstica análogamente a los de la violencia política, pero despolitizando ambas, por supuesto. Como demuestra Judith Herman, en Trauma and Recovery (1992), las consecuencias de la violencia y el terror político son muy similares para las víctimas (Fortune y Adams,2006:2505) por lo que no es de extrañar que en torno al terrorismo político y a la violencia doméstica se construyera, a principios de los años dos mil, el consenso social.

${ }^{9}$ Por emergencia entendemos un tratamiento despolitizado y excepcional de las cuestiones, ya se trate de la extranjería, el terrorismo o la violencia doméstica. (Marugán y Vega,2002:428).
} 
a gestionar como emergencia y como tal incluyó planes ${ }^{10}$, estructuras y acuerdos que permitieron comprometer los esfuerzos del gobierno con entidades privadas coordinando actuaciones ${ }^{11}$ para evitar los asesinatos, pero no atacaron ni la discriminación, ni el androcentrismo, elementos desencadenantes de las agresiones.

La Resolución A4-0250/97 del Parlamento europeo sobre una campaña europea sobre tolerancia cero ante la violencia contra las mujeres que instaba a los Estados miembros a que establecieran una legislación específica fuera del Código penal para proteger a las víctimas de la violencia por razones de sexo, tuvo su concreción en España en el I Plan Integral Contra la Violencia Doméstica (1998-2000) ${ }^{12}$. El foco de actuación se había reducido al ámbito doméstico y de este modo la violencia contra las mujeres se parcela, de manera que solo se saca a la luz la violencia más disfuncional, la doméstica, la que puede quebrar los entornos familiares o llegar a poner en cuestión la familia, la convivencia y la propia reproducción social.

Con la aparente inocuidad del cambio nominal de «violencia contra las mujeres» a «violencia doméstica» se había producido un desplazamiento discursivo. Lo que por un lado hizo público el fenómeno, por otro, paradójicamente, redujo la violencia sobre las mujeres a una sola de sus manifestaciones - la que se ejerce por parte de los compañeros sentimentales-. «El concepto "violencia doméstica" establece fronteras innecesarias entre el hogar y el espacio público, la calle, en el que también se ejerce gran cantidad de violencia contra las mujeres» (Miranda, 2006: 2486). Fuera quedaba el acoso sexual en el trabajo, la violencia sexual, la utilización de la violación como arma de guerra o la trata de mujeres por ejemplo.

En un tiempo donde, después del 11 de septiembre, el paradigma dominante era el de la seguridad, desde el Estado se articulan una serie de dispositivos jurídicos, penales y asistenciales para evitar los asesinatos de mujeres. Entre ellos se aprobó la Ley 27/2003, de 31 de julio, reguladora de la Orden de Protección de las Víctimas de Violencia Doméstica, así como medidas específicas dentro de la Ley Orgánica 11/2003, de 29 de septiembre. También se adoptaron Medidas Concretas en Materia de Seguridad Ciudadana, Violencia Doméstica e Integración Social de los Extranjeros.

\footnotetext{
${ }^{10}$ Las políticas públicas frente a la violencia se articularon en torno al I Plan Integral contra la Violencia Doméstica que contó con un presupuesto de 8.004 millones de pesetas -47.643 euros- y estaba compuesto por 57 medidas enmarcadas en seis áreas: legislación y práctica jurídica, sensibilización y prevención, educación y formación, recursos sociales y sanidad.

${ }^{11}$ Se dio mucha relevancia a la coordinación y por ello se hablaba de Planes "integrales", lo que habría sido deseable puesto que esta coordinación no se consiguió.

${ }^{12}$ Al que le seguiría un II Plan Integral contra la Violencia Doméstica (2001-2004).
} 
Por otra parte, bajo el paraguas de «la domesticidad» se oculta la dominación de los hombres sobre las mujeres (Marugán, 2010). La expresión «doméstica», presenta un tono eufemístico que oscurece el género de la víctima y del perpetrador de modo que no resulta clara la imposición de dominio masculino contra una pareja femenina (Fortune y Adams, 2006: 2504).

Para evitar esta labor de ocultamiento se recurrió, en algún momento, al uso de la denominación «violencia familiar». Concepto creado en el I Congreso de Organizaciones Familiares celebrado en Madrid, en 1987. Por «violencia familiar» se define «toda aquella acción u omisión de uno o varios miembros de la familia que dé lugar a tensiones, vejaciones u otras situaciones similares en los distintos miembros de la misma» (Ministerio del Interior, 1991). Obviamente, el término «violencia familiar» implica a todos los miembros de la familia, de modo que en principio introduce mayor confusión. Para evitar la misma se dio paso al término «violencia de género». Con el mismo se pretendía señalar el origen de la misma. «Probablemente la principal ventaja de hablar de violencia de género sea caracterizar este tipo de violencia por su conexión con el despliegue efectivo de diferentes modelos de género y por las relaciones y procesos que entre ellos se van dando, pues así resalta que es una violencia ejercida y desplegada en los procesos de relaciones de poder que son las relaciones de género» (García Selgás y Casado, 2010:112).

La exposición de motivos de la Ley Orgánica 1/2004, de 28 de diciembre, de Medidas de Protección Integral contra la Violencia de Género, indica que «la violencia de género es el símbolo más brutal de la desigualdad existente en nuestra sociedad», sin embargo, la ocupación en el imaginario de la violencia de género como "violencia doméstica", ha supuesto una rémora y ha conllevado un desplazamiento metonímico de la parte por el todo. Podrían servir para comprender este proceso las explicaciones que ofrece Víctor W. Turner en El proceso ritual. Según este autor el simbolismo puede ser utilizado como clave para comprender la estructura social. Los símbolos -y en este caso el lenguaje puede entenderse como tal- no son únicamente una serie de clasificaciones cognitivas para ordenar el universo, sino también dispositivos evocadores que suscitan, encauzan y domestican las emociones fuertes como el odio, el miedo, el afecto o el dolor y cada elemento simbólico guarda relación con algún elemento empírico de la experiencia" (Turner, 1988: 53). Lo lamentable es la cantidad de experiencia acumulada que la población tiene sobre la violencia conyugal, al ser la expresión más numerosa de todos los tipos de violencia que se ejerce sobre las mujeres en el Estado español.

El hecho es que la adopción durante más de cinco años del concepto «violencia doméstica» ha tenido importantes efectos. Así, aunque la Ley 1/2004 utiliza el concepto "violencia de género" -repolitizando esta cuestión- sólo tiene como objetivo actuar "contra la violencia que, como manifestación de la discriminación, la situación de desigualdad y las relaciones de poder de los hombres sobre las mujeres, se ejerce 
sobre éstas por parte de quienes sean o hayan sido sus cónyuges o de quienes estén o hayan estado ligados a ellas por relaciones similares de afectividad, aún sin convivencia" (Art. 1.1). De este modo deja fuera del ámbito de su intervención el acoso sexual en los ámbitos laborales o la violencia sexual y no tiene en cuenta la dominación masculina que sigue plenamente vigente en todos los ámbitos de la vida pública. La Ley solo atiende a esa dominación cuando se da en el espacio privado, lo cual también es paradójico y viene a reforzar las ideas de aquellos que piensan que el lugar de las mujeres sigue estando en su casa.

\section{BIBLIOGRAFÍA}

BROWMILLER, S (1975): Contra nuestra voluntad, Barcelona, Planeta.

BOURDIEU, P. (1982): Ce que parler veut dire, l'économie des echanges linguistiques, PARIS, FAYARD.

CORSI, J. (1995): Violencia familiar. Una mirada interdisciplinar sobre un grave problema social, Barcelona, Paidos.

DIO BLEICHMAR, E. (2001): "Efectos tempranos y tardíos de la violencia de género", en Bernárdez, A (Ed.), Violencia de género y sociedad: Una cuestión de poder, Madrid, Instituto de Investigaciones Feministas y Ayuntamiento de Madrid, 47-62

FERREIRA, G. (1992): Hombres violentos, mujeres maltratadas, Buenos Aires, Editorial Sudamericana.

FORTUNE, M.M y ADAMS, C.J. (2006): "Violencia doméstica", en Kramarae, Ch y Dale Spender D (eds). Enciclopedia Internacional de las Mujeres. Edición especial para el ámbito hispano hablante, volumen 5. Q-Z (Rosa García Rayego y Gloria Nielfa Cristobal (editoras de la Edición española), Madrid, Editorial Síntesis, 2504- 2510.

GARCÍA SELGAS, F (2009): "La investigación social sobre violencia de género: una propedéutica", en Miranda, $\mathrm{M}^{\mathrm{a}} \mathrm{J}$, Martín, M ${ }^{\mathrm{a}} \mathrm{T}$ y Marugán, B, (eds), Amor, Razón y Violencia, Madrid, Los libros de La Catarata.

GARCÍA SELGAS, F Y CASADO APARICIO, E (2010): Violencia en la pareja: género y vínculo, Madrid, Talasa Ediciones.

GELLES, R. J. y STRAUS, M. A. (1988): Intimate violence, New York, Simon \& Schuster.

KAUFMAN J, ZIGLER E, (1987): "Do abused children become abusive parents? Am J Orthopsychiatry. Apr,57(2),186-92

KRAMARAE, Ch y SPENDER, D (eds): (2006) Enciclopedia Internacional de las Mujeres. Edición especial para el ámbito hispano hablante, volumen 5. Q-Z (Rosa García Rayego y Gloria Nielfa Cristobal (editoras de la Edición española), Madrid, Editorial Síntesis. 
MARUGÁN PINTOS, B, (2001): "Análisis de los discursos del movimiento Feminista contra las agresiones a las mujeres. Una mirada autoreflexiva", en Feminismo.es... y será, Actas de las Jornadas Feministas, Córdoba, Universidad de Córdoba, Córdoba, 369-376.

MARUGÁN PINTOS, B y VEGA SOLÍS, C. (2002): "Gobernar la violencia: apuntes para un análisis de la articulación del patriarcado", en la Revista Política y Sociedad vol. 39 n. 2 (2002), UCM, 415-436.

MARUGAN PINTOS, B y VEGA SOLIS, C. (2003): "Acción feminista y gubernamentaliad", Revista Contrapoder o http://revistacontrapoder.net/article.php3?id article $=21$. La versión alemana del mismo forma parte del libro de Pieper, M y Rodríguez, G (2003) Gouvernementalität, Frankfurt, Campus.

MIRANDA LÓPEZ, M. (2002): "Violencia contra las mujeres y las niñas," en Coeducar en los afectos, Madrid, Federación de Enseñanza de CC.OO, 19-26.

PATEMAN, C. (1995): El contrato sexual, Madrid, Anthropos.

REINARES, F. (1998): "Violencia", en Giner, S, Lamo de Espinosa, E y Torres, c (eds), Diccionario de sociología, Madrid, Alianza Editorial.

RUBIN, G (1975): "The traffic in women. Notes on the political economy of sex", en Reitre, Rayna (ed), Toward an Antropology of women, N.Y, Monthly Review Press, 1975, 157-210.

SANCHEZ VIDANES, M.C y CARRASCO SERRANO, F. (2001): "El derecho ante los malos tratos a las mujeres", en Feminismo.es...y será, Universidad de Córdoba, Córdoba, 131-140.

SCHWARTZ COWAN, R, (2011): "La 'Revolución Industrial' en el hogar: Tecnología doméstica y cambio social en el Siglo XX", en Carrrasco, C, Borderías, C y Torns, T (2011), El trabajo de cuidados. Historia, teoría y política, Madrid, Los Libros de la Catarata,97-121.

TURNER, V.W. (1988): El proceso ritual, Madrid, Taurus.

WALTER, L. (1979): Battered woman, New York, Harper y Row Publishers. 